\title{
Amyloid A as a biomarker for preeclampsia
}

\author{
Original \\ Mohamed Ahmed Hassan El-Kady ${ }^{1}$, Dina Yahia Mansour Ali', Noha Hussein \\ Article \\ Boshnak', Salma Ali Mustafa Abdulqader Ahmed ${ }^{1}$
}

${ }^{1}$ Department of Obstetrics and Gynecology, ${ }^{2}$ Department of Clinical Pathology, Faculty of Medicine, Ain-Shams University, Cairo, Egypt

\begin{abstract}
Aim:To investigate and evaluate serum levels of amyloid A in PET and healthy gestations, to help in detection of a correlation between PET and serum levels of amyloid A.

Methodology: A randomized case control research study conducted at Ain-Shams University Maternity Hospital, Cairo, Egypt in a period of 6 months. There was 102 study subjects in the total cohort ; 51 cases diagnosed with preeclampsia and 51 study subjects with normal gestations as a control research group.

Results: The median serum level of amyloid-A was statistically significantly higher in women of PE research group in comparison to women of the control research group ( $p$ value $<0.001)$. Furthermore, the fetal amyloid A was not statistically significantly different between both research groups. Among women of research group I (PE Group), there was no statistical significant correlation between serum amyloid-A level and systolic BP, diastolic $\mathrm{BP}$, mean arterial BP ( $p$ values $=0.128,0.112,0.119$ consecutively). However there was statistical significant correlation between serum amyloid A and serum ALT, serum AST, serum creatinine, GA at delivery and birth weight ( $p$ values $=<0.001,<0.001,<0.001,<0.001,0002,<0.001$ consecutively).

Conclusion: Serum amyloid-A 1 is a promising biomarker in detectability and predictability of the pathological development of preeclampsia. Future research studies should be conducted in a multicentric fashion.
\end{abstract}

Key Words: Amyloid A, biomarker, preeclampsia

Received: 1 October 2019, Accepted: 2 October 2019

Corresponding Author: Salma Ali Mustafa Abdulqader Ahmed, Department of Obstetrics and Gynecology, Faculty of Medicine, Ain-Shams University, Cairo, Egypt,Tel.: 01090847409, E-mail: salmaali86666@gmail.com

ISSN: 2090-7625, February 2020, Vol.10, No.1

\section{INTRODUCTION}

Hypertensive disorder is one of the most frequent gestational medical disorders and the incidence of hypertensive diseases has a range from $5 \%$ to $6 \%$ in all types of gestations. Hypertensive diseases in conjunction with bleeding and infectious diseases are the corner stone causes for the bulk of maternal morbidity and mortality during gestation. Additionally, hypertension accounts for around $16 \%$ of maternal mortalities in developed nations $^{[1,2]}$.

Serum amyloid-A levels rise in a significant manner as pathophysiological response to infectious viral and bacterial insults, tumor enlargement and physical strain. Serum amyloid A may rise up to 1000 -fold and could reach to $500-1000 \mu \mathrm{g} / \mathrm{mL}$. Amyloid A is synthesized by hepatic cells as an inflammatory response to cytokines ${ }^{[3,4]}$.

It has been reported that additionally amyloid $\mathrm{A}$ in serum is manufactured by various cell types such as fibroblasts, macrophages and fat cells. Restricted number of research studies have assessed and evaluated amyloid A levels in serum of gestations suffering the development of $\mathrm{PET}^{[5,6]}$

\section{AIM OF THE WORK}

To investigate and evaluate serum levels of amyloid A in PET and healthy gestations, to help in detection of a correlation between PET and serum levels of amyloid A.

\section{PATIENTS AND METHODS}

A randomized prospective case control research study conducted at Ain-Shams University Maternity Hospital, Cairo, Egypt, in a period of 6 months. There was 102 study subjects in the total cohort ; 51 cases diagnosed with preeclampsia and 51 study subjects with normal gestations as a control research group.

Inclusive research criteria were as follows: cases age range from 18-35 years. Singleton gestations, no other medical disorders with pregnancy (e.g DM, SLE, bronchial asthma, chronic hypertension), absence 
of bleeding issues (e.g antepartum haemorrhage), absence of placental abnormalities (e.g placenta previa, morbidly adherent placenta), no congenital fetal malformation (e.g neural tube defects), no fetal macrosomia (EFW $>4 \mathrm{Kg}$ ), no polyhydramnios, range of gestational age 35-40 weeks. Caesarean section delivery performed for obstetric indications.

Exclusive research criteria were as follows: Cases that refused participation in the research study, diabetes mellitus (DM) or other medical disorders (as chronic hypertension, bronchial asthma, cardiac disease) associated with PET, preterm or post term gestations, early rupture of membrane (PROM), antepartum haemorrhage (revealed or concealed), multi fetal gestations, congenital fetal malformation (e.g. neural tube defects, congenital heart disease), gestations with coagulative disorders (e.g. prolonged PT, PTT), hepatic or renal disorders. (e.g. affection of renal or hepatic functions e.g. hepatitis, renal stones), body mass index (BMI) more than 35, immunological disorders (e.g. SLE, hashimoto thyroiditis), cases on drugs (e.g. anti-coagulant and anti-platelet such as acetyl salicylic acid, heparin), normal vaginal delivery or women in active labour ; since active labor could trigger an inflammatory cascade process and therefore could alter the research study findings, therefore elective caesarean deliveries were chosen.

The following steps were performed in study procedures: PET clinical diagnosis was considered when $\mathrm{BP}$ of $>140 / 90 \mathrm{mmHg}$ is confirmed by two measurements at 6 hours-time interval and in the existence of protein in urine $(>300 \mathrm{mg} / \mathrm{L})$ in a 24 hours urine specimen or dipstick test $1+$.

Severe PET was clinically described as a BP measurement of $>160 / 110 \mathrm{mmHg}$, protein/ creatinine in urine $(>5 \mathrm{~g} / 24 \mathrm{~h})$ dipstick test $3+$, oliguria $(<500 \mathrm{~mL} / 24 \mathrm{~h})$, visual disorders, headache, pain in the epigastrium, serum creatinine raised serum levels, thrombocytopenia $(<100000 / \mathrm{mm} 3)$, elevated liver enzymes (SGOT and SGPT) and/or abnormal peripheral smear, clinical existence of pulmonary edema, and IUGR Coexisting with umbilical artery

Doppler abnormal indices or oligohydramnios. Sociodemographic features, full medical history and laboratory indices of gestations including $\mathrm{CBC}$, $\mathrm{BUN}$, serum creatinine, SGOT and SGPT were gathered and tabulated.

Sonographic fetal data and blood gas results were recorded. As labor could trigger an inflammatory response and thereby may affect the results. Women who gave birth through elective caesarean section were selected.

Since the time interval between blood sample collection and CS performance could influence the results; $8 \mathrm{~mL}$ of blood sample was collected from research study subjects within two hours before CS performance for the analysis of serum levels of amyloid-A and $8 \mathrm{~mL}$ of blood sample were collected from the placental side of the cord after cutting the umbilical cord at delivery to analyze serum levels of amyloid-A in cord blood.

The obtained blood sample has been centrifuged at a rate of $4500 \mathrm{rpm}$ for $15 \mathrm{~min}$ and the serum were stored at $-80^{\circ} \mathrm{C}$ till the time of lab processing. Amyloid-A serum level analysis was performed by the Invitrogen human serum amyloid-A enzyme linked immunosorbent assay (ELISA) kit (Invitrogen Corporation, Camarillo, CA, USA).

The detection limit of the assay was established at $4 \mathrm{ng} / \mathrm{mL}$ (ELISA) is a method of target antigen or antibody capture in samples using a specific antibody (or antigen) and of target molecule detection/quantitation using an enzyme reaction with its substrate. Antibodies are bound to a plastic surface, a sample is added and if antigens are present they will stick to the antibodies.

\section{STATISTICAL ANALYSIS:}

The collected data will be revised, coded, tabulated and introduced to a PC using Statistical Package for Social Science (*SPSS 20.0.1 for windows; SPSS Inc, Chicago, IL, 2001) quantitative variables are expressed as mean and $\mathrm{SD}$, or Median and interquartile range (IQR) according to distribution of data.

Qualitative variables are expressed as frequencies and presents. Student $t$ test and Mann Whitney Test will be used to compare a continuous variable between two study groups. Chi square test will be used to examine the relationship between categorical variables. A $p$-value $<0.05$ was considered statistically significant.

\section{RESULTS}

A total of 51 pregnant women with preeclampsia were included as group I (PE Group) along with 51 normotensive non-proteinuric pregnant women as group II (Control Group).There were no statistical significant differences between women of both groups regarding age, parity and BMI BMI $(p$ values $=0.098,0.794,0.065$, consecutively $)$ (Table 1).

The median serum level of amyloid-A was statistically significantly higher in women of PE research group in comparison to women of the control research group ( $p$ value $<0.001)$. Furthermore, the fetal amyloid-A was not statistically significantly different between both research groups (Table 2). 
Table 1: Difference between groups regarding initial characteristics

\begin{tabular}{|c|c|c|c|c|}
\hline & $\begin{array}{c}\text { Group I } \\
\text { [PE Group] } \\
(\mathrm{n}=51)\end{array}$ & $\begin{array}{c}\text { Group II } \\
\text { [Control Group] } \\
(\mathrm{n}=51)\end{array}$ & $\begin{array}{c}\text { MD/MedD } \\
(95 \% \mathrm{CI})\end{array}$ & $P$ \\
\hline Age (years) & $22-37$ & $23-36$ & 1.43 & 0.0981 \\
\hline Range & $29.18 \pm 4.11$ & $30.61 \pm 4.53$ & $(-0.27$ to 3.12$)$ & NS \\
\hline \multicolumn{5}{|l|}{ Mean $\pm \mathrm{SD}$} \\
\hline Parity & $0-4$ & $0-4$ & 0 & 0.7942 \\
\hline Range & $2(0-3)$ & $2(1-3)$ & $(0-1)$ & NS \\
\hline \multicolumn{5}{|l|}{ Median (IQR) } \\
\hline BMI (kg/m2) & $18.1-42.6$ & $18.3-42.9$ & 2.4 & 0.0651 \\
\hline Range & $30.82 \pm 6.71$ & $28.42 \pm 6.25$ & $(-4.95$ to 0.15$)$ & NS \\
\hline Mean $\pm \mathrm{SD}$ & & & & \\
\hline
\end{tabular}

SD standard deviation

IQR interquartile range

BMI body mass index

MD $(95 \% \mathrm{CI})$ mean difference and its $95 \%$ confidence interval

MedD $(95 \% \mathrm{CI})$ median difference and its $95 \%$ confidence interval

Data presented as range, mean $\pm \mathrm{SD}$; or range, median (IQR)

1 Analysis using independent student's t-test

2 Analysis using Mann-Whitney’s U-test

NS non-significant

Table 2: Difference between groups regarding serum amyloid-A

\begin{tabular}{|c|c|c|c|c|}
\hline & $\begin{array}{c}\text { Group I } \\
\text { [PE Group] } \\
(\mathrm{n}=51)\end{array}$ & $\begin{array}{c}\text { Group II } \\
\text { [Control Group] } \\
(n=51)\end{array}$ & $\begin{array}{c}\text { MedD } \\
(95 \% \mathrm{CI})\end{array}$ & $P=1$ \\
\hline \multicolumn{5}{|c|}{$\begin{array}{l}\text { Maternal Amyloid-A } \\
(\mathrm{ng} / \mathrm{dl})\end{array}$} \\
\hline Range & $10.7-129.1$ & $4.5-34.2$ & -46.7 & $<0.001$ \\
\hline Median (IQR) & $68.1(35.8-95.1)$ & $16.6(11.4-26.4)$ & $(-62.3$ to -31.2$)$ & HS \\
\hline \multicolumn{5}{|l|}{ Fetal } \\
\hline Range & $2.3-65.6$ & $2.7-59.8$ & -5.325 & \\
\hline Median (IQR) & $28.5(18.3-38.2)$ & $21.6(15.4-42.6)$ & $(-2.4--7.7)$ & 0.516 \\
\hline
\end{tabular}

IQR interquartile range

MedD (95\% CI) median difference and its 95\% confidence interval

Data presented as range, median (IQR)

1 Analysis using Mann-Whitney’s U-test

HS highly significant 


\section{DISCUSSION}

Preeclampsia is a common complication of gestation and remains a chief cause of maternal and fetal mortality. The clinical symptoms of preeclampsia are caused by widespread endothelial dysfunction proposed to be a part of an exaggerated maternal inflammatory response to pregnancy.

Serum amyloid A1 (SAA1) is an acute response protein, which is chiefly synthesized by the liver, during the process of infection. On the other hand, it remains unidentified whether SAA1 could be created in human fetal membranes where it is capable to trigger events pertinent to labor commencement ${ }^{[7,8]}$.

Serum amyloid A like CRP is an inflammation marker produced in the liver. Their hepatic synthesis is induced by cytokines such as IL-6 and TNF alpha. SAA can affect the onset and progress of endothelial dysfunction by inflammation, however there are no reports on the relation between the SAA and preeclampsia ${ }^{[9,10]}$.

Interestingly a prior research study have shown a significant difference between the maternal blood and umbilical cord blood amyloid A serum levels both in the preeclampsia and control. Contradicting to the current research study a prior research group has revealed that plasma levels of serum amyloid A protein were not elevated in women with preeclampsia in comparison to women with normal gestation ${ }^{[11,12]}$.

\section{CONCLUSION}

Serum amyloid A 1 is a promising biomarker in detectability and predictability of the pathological development of preeclampsia. Future research studies should be conducted in a multicentric fashion.

\section{CONFLICT OF INTEREST}

There are no conflict of interests.

\section{REFERENCES}

1. Mol BW, Roberts CT, Thangaratinam S, Magee LA, de Groot CJ, Hofmeyr GJ. (2015): Pre-eclampsia. Lancet; 387: 999-1011.

2. Anthony D, McQualter JL, Bishara M, Lim EX, Yatmaz S, Seow HJ, Hansen M, Thompson M, Hamilton JA, Irving LB, Levy BD, Vlahos R, Anderson GP, Bozinovski S. (2014): SAA drives proinflammatory heterotypic macrophage differentiation in the lung via CSF-1R-dependent signaling. FASEB J; 28: 3867-3877.

3. Enkhmaa D, Wall D, Mehta PK, Stuart JJ, RichEdwards JW, Merz CN, Shufelt C. (2016): Preeclampsia and vascular function: A window to future cardiovascular disease risk. J. Women's Health; 25, 284-291.

4. Hansen MT et al. (2015): A link between inflammation and metastasis: serum amyloid A1 and A3 induce metastasis, and are targets of metastasis-inducing S100A4. Oncogene; 34: 424-435.

5. Ayansina D, Black C, Hall SJ, Marks A, Millar C, Prescott GJ, Wilde K, Bhattacharya S. (2016): Long term effects of gestational hypertension and pre-eclampsia on kidney function: Record linkage study. Pregnancy Hypertens; 6: 344-349.

6. Boeldt DS, Bird IM. (2017): Vascular adaptation in pregnancy and endothelial dysfunction in preeclampsia. J. Endocrinol; 232: R27-R44.

7. Ye, RD and Sun L. (2015): Emerging functions of serum amyloid $\mathrm{A}$ in inflammation. $\mathrm{J}$ Leukoc Biol 98, 923-929.

8. Chaiworapongsa $\mathrm{T}$, Chaemsaithong $\mathrm{P}$, Yeo L, Romero R. (2014): Pre-eclampsia part 1: Current understanding of its pathophysiology. Nat. Rev. Nephrol; 10: 466-480.

9. Yuee Ling, Jie Su, Jie Lin \& Sumei Wang (2019) Screening of serum biomarkers of preeclampsia by proteomics combination with bioinformatics, Hypertension inPregnancy, 38:3, 184192-, DOI: 10.108010641955.2019.1640246/

10. Chen M, Zhou H, Cheng N, Qian F, Ye RD. (2014): Serum amyloid A1 isoforms display different efficacy at Toll-like receptor 2 and formyl peptide receptor 2. Immunobiology; 219: 916-923.

11. Harmon AC, Cornelius DC, Amaral LM, Faulkner JL, Cunningham MW, Jr, Wallace K, LaMarca B. (2016): The role of inflammation in the pathology of preeclampsia. Clin. Sci; 130: 409-419.

12. ConnollyM,VealeDJ,FearonU(2011):Acuteserum amyloid A regulates cytoskeletal rearrangement, cell matrix interactions and promotes cell migration in rheumatoid arthritis. Annals of the Rheumatic Diseases; 70: 1296-1303. 